\title{
Physical properties of madocite: A quaternary chalcogenide with very low thermal conductivity
}

\author{
Kaya Wei ${ }^{1}$, Joshua Martin ${ }^{2}$, Satofumi Maruyama ${ }^{3}$, Takao Mori ${ }^{3}$ and George S. Nolas ${ }^{1, *}$ \\ ${ }^{1}$ Department of Physics, University of South Florida, Tampa, FL 33620 \\ ${ }^{2}$ Material Measurement Laboratory, National Institute of Standards and Technology, \\ Gaithersburg, MD 20899 \\ ${ }^{3}$ National Institute for Materials Science, MANA, Tsukuba, 305-0044 Japan
}

\begin{abstract}
The madocite $\mathrm{Pb}_{17}\left(\mathrm{Sb}_{0.75} \mathrm{As}_{0.25}\right)_{16} \mathrm{~S}_{41}$ was synthesized by reaction of binary compounds and densified using hot pressing. The optical band-gap, thermal stability, and temperature-dependent heat capacity and thermal conductivity are reported for the first time. The thermal properties are evaluated using the Debye model. The findings in this work lay the foundation for a more detailed understanding of the physical properties of this material, and is part of the continuing effort in investigating materials with new compositions that possess low thermal conductivity for potential low-cost thermal barrier coating and energy-related applications.
\end{abstract}

*E-mail: gnolas@usf.edu 


\section{INTRODUCTION}

Multinary chalcogenides come in several different structure types, each with unique properties that are of interest for specific applications. For example, kesterite compositions are of interest as solar cell materials due to the characteristic of the band structure.[1] Another multinary chalcogenide $\mathrm{Ag}_{2} \mathrm{HgPbSe}_{4}$ has been shown to be a topological insulator with optimal negative crystal field splitting and inverted band gap.[2] Very recently certain multinary chalcogenides have shown to possess promising thermoelectric properties mainly due to the intrinsically poor thermal conductance.[3-6] As a solid-state method of energy conversion, thermoelectricity plays an important role in both power generation and cooling applications and requires low thermal conductivity, $\kappa$, to reach a high energy conversion efficiency.[7] Such requirement is also essential in rewriteable devices and thermal barrier materials.[8-10]

Different compositions of multinary chalcogenides result in different physical properties, making these materials of great interest for a variety of applications. It is therefore of interest to explore the physical properties of different multinary chalcogenides. One such example, and the focus of this report, is $\mathrm{Pb}_{17}\left(\mathrm{Sb}_{0.75} \mathrm{As}_{0.25}\right)_{16} \mathrm{~S}_{41}$, a multinary chalcogenide with the madocite crystal structure. This material possesses a relatively complex crystal structure with a large unit cell, a criterion that typically results in low thermal conductivity, $\kappa$. We therefore synthesized and investigated the physical and chemical properties of $\mathrm{Pb}_{17}\left(\mathrm{Sb}_{0.75} \mathrm{As}_{0.25}\right)_{16} \mathrm{~S}_{41}$. The thermal properties are understood using the Debye model, [11] while the thermal stability and optical properties are investigated in order to begin the fundamental understanding of this material, as well as provide the basis for potential future investigations. 


\section{EXPERIMENT}

High Purity $\mathrm{Pb}_{17}\left(\mathrm{Sb}_{0.75} \mathrm{As}_{0.25}\right)_{16} \mathrm{~S}_{41}$ was prepared by reaction of the binaries; $\mathrm{PbS}$ powder $(99.9 \%$, Alfa Aesar), $\mathrm{As}_{2} \mathrm{~S}_{3}$ powder (99.9\%, Alfa Aesar), and $\mathrm{Sb}_{2} \mathrm{~S}_{3}$ powder (99.8 \%, Alfa Aesar) which were loaded into a silica ampoule in a $\mathrm{PbS}: \mathrm{As}_{2} \mathrm{~S}_{3}: \mathrm{Sb}_{2} \mathrm{~S}_{3}$ atomic ratio of 8.5:1:3 inside a $\mathrm{N}_{2}$ filled glove box to minimize exposure of the reactants to air. ${ }^{1}$ The ampoule was sealed in a quartz tube, heated to $673 \mathrm{~K}$ and held at this temperature for four days. The furnace was subsequently turned off and the product slowly cooled to room temperature. The product was then ground into fine powders, cold pressed into a pellet and annealed at $623 \mathrm{~K}$ for two weeks to promote homogeneity. The resulting pellet was then ground into fine powders (325 mesh) inside a glove box and loaded into a graphite die for hot pressing. Densification was accomplished by hot pressing at $623 \mathrm{~K}$ and $160 \mathrm{MPa}$ for 3 hours under $\mathrm{N}_{2}$ flow. The density of the hot-pressed pellet was determined by measurement of the dimension and weight and indicated that a high density polycrystalline specimen $(98 \%)$ was obtained.

X-ray diffraction (XRD) and energy dispersive spectroscopy (EDX) were used to examine the purity and homogeneity of the madocite compound. Powder XRD data were collected with a Bruker D8 Focus diffractometer in Bragg-Brentano geometry using $\mathrm{Cu} \mathrm{K} \alpha$ radiation and a graphite monochromator. EDX analyses was accomplished with an Oxford INCA X-Sight 7582M equipped scanning electron microscope (SEM, JEOL JSM-6390LV). The homogeneity of the specimen was investigated from twelve EDX data sets obtained from random positions across the dense pellet, as well as from XRD analyses.

\footnotetext{
${ }^{1}$ Certain commercial equipment, instrumentation, or materials are identified in this document. Such identification does not imply recommendation or endorsement by the National Institute of Standards and Technology, nor does it imply that the products identified are necessarily the best available for the purpose.
} 
The densified pellet was cut into $2 \mathrm{~mm} \times 2 \mathrm{~mm} \times 5 \mathrm{~mm}$ parallelepipeds using a wire saw for temperature dependent four-probe resistivity, $\rho$, and steady-state $\kappa$ measurements from 12 to 300

$\mathrm{K}$. The measurements were conducted in a custom designed radiation-shielded vacuum probe with an experimental uncertainty of $4 \%$ for $\rho$ and $8 \%$ for $\kappa \cdot[12,13]$ High temperature thermal diffusivity measurements, on a separate part of the pellet, were conducted under flowing Ar with an Anter flashline system. The uncertainty in the thermal diffusivity measurements was $5 \%$. High temperature resistivity measurements were conducted by the four-probe method with an ULVAC system with an experimental uncertainty of 5\%. Good agreement at room temperature between the two measurements, corroborate the homogeneity of the polycrystalline specimen. Optical absorption spectra were obtained using a UV-Vis spectrometer (Jasco, V-670 Spectrophotometer). Differential thermal analysis (DTA) and thermal gravimetric analysis (TGA) (TA Instruments Q600) were used under $\mathrm{N}_{2}$ to determine the decomposition temperature. Heat capacity was measured using a commercial Quantum Design Physical Property Measurement System in the temperature range of $5 \mathrm{~K}<\mathrm{T}<310 \mathrm{~K}\left(1.3 \times 10^{-5} \mathrm{~Pa}\right)$. Thermal coupling between the specimen and the specimen platform was achieved via Apiezon® $\mathrm{N}$ grease. Prior to each specimen measurement, the specimen puck, the specimen platform, and the Apiezon ${ }^{\circledR} \mathrm{N}$ grease specifically used in the measurement were thoroughly characterized in a separate addenda measurement. The uncertainty in the measurement was $7 \%$ throughout the measured temperature range.

\section{RESULTS AND DISCUSSION}


$\mathrm{Pb}_{17}\left(\mathrm{Sb}_{0.75} \mathrm{As}_{0.25}\right)_{16} \mathrm{~S}_{41}$ forms in an orthorhombic symmetry with space group $P b 2 a .[14,15]$ Figure 1 shows the powder XRD pattern with a schematic of the atomic structure as the inset. This material has a large, 74 atom, unit cell. Our EDS analyses agree with the powder XRD data and indicate a phase-pure composition. Figure 2 shows DTA and TGA data indicating an exothermic decomposition temperature at $668 \mathrm{~K}$ with a change in mass at the same temperature. Above this temperature madocite decomposes to stibnite $\left(\mathrm{Sb}_{2} \mathrm{~S}_{3}\right)$ and sulfide $\left(\mathrm{S}_{2} \mathrm{O}_{3}\right)$, as observed by XRD of the product after DTA/TGA. Figure 3 shows room temperature UV-Vis spectra and indicates an optical band-gap of $1.3 \mathrm{eV}$.

Isobaric specific heat, $C_{\mathrm{p}}$, measurements from $5 \mathrm{~K}$ to $310 \mathrm{~K}$ with $1.6 \mathrm{mg}$ of specimen are shown in Figure 4. The inset in Figure 4 shows $C_{\mathrm{p}} / T$ versus $T^{2}$ data below $10 \mathrm{~K}$. The solid line in the inset is a fit to the data of the form $C_{\mathrm{p}} / T=\gamma+\beta T^{2}$, where $\gamma$ is the Sommerfeld coefficient of the electronic contribution to the specific heat and $\beta$ is the coefficient of the lattice contribution.[16,17] From this fit we obtain $\gamma=361 \mathrm{~mJ} \mathrm{~mol}^{-1} \mathrm{~K}^{-2}$ and $\beta=51 \mathrm{~mJ} \mathrm{~mol}^{-1} \mathrm{~K}^{-4}$, values that are relatively high compared with that of metals but lower than that of oxides and other wide band gap semiconductors.[18-20]

Using the relation

$$
\theta_{D}=\left(\frac{12 \pi^{4} R n_{a}}{5 \beta}\right)^{1 / 3},
$$

where $R$ is the molar gas constant and $n_{\mathrm{a}}$ is the number of atoms per formula unit ( $n_{\mathrm{a}}=74$ for $\left.\mathrm{Pb}_{17}\left(\mathrm{Sb}_{0.75} \mathrm{As}_{0.25}\right)_{16} \mathrm{~S}_{41}\right)$, we estimate $\theta_{D}$ to be $141 \mathrm{~K}$. In the Debye model, $\theta_{D}$ is linearly proportional to the Debye cutoff frequency $\omega_{D}, \theta_{D}=\omega_{D} * \hbar / k_{B}$, and is proportional to the average 
group velocity $v_{g}\left(\omega_{D} \approx v_{g} * K_{D}\right.$ with $K_{D}$ being the maximum allowable wave vector).[21] Since $v_{g}$ decreases with decreasing hardness and bonding energy, a small $v_{g}$ can be an indication of relatively low hardness and loose bonding, [22] also suggesting intrinsically low $\kappa$ for this material. The relatively week $\mathrm{Pb}-\mathrm{S}$ bonds [14] presumably result in a relatively small $v_{g}$, as estimated from our modeling, and therefore relatively low $\kappa_{\mathrm{L}}$ values.

Figure 5 shows temperature dependent $\kappa$. The $\rho$ values were very high in the entire temperature range of our measurements, $\rho>1 \mathrm{Ohm}-\mathrm{m}$, therefore $\kappa_{\mathrm{L}}$, the lattice part of $\kappa$, is presumed to be the dominant contribution to $\kappa . \kappa$ is below $1.0 \mathrm{~W} / \mathrm{m}-\mathrm{K}$ from $100 \mathrm{~K}$ up to the highest measured temperature. The solid line in Figure 5 is a theoretical fit to the data using the Debye approximation [11]

$$
\kappa_{L}=\frac{k_{B}}{2 \pi^{2} v}\left(\frac{k_{B} T}{v}\right)^{3} \int_{0}^{\theta_{D} / T} \frac{x^{4} e^{x}}{\tau_{C}^{-1}\left(e^{x}-1\right)^{2}} d x
$$

where $x=\hbar \omega k_{B}^{-1} T^{-1}$ is dimensionless, $\omega$ is the phonon frequency, $v$ is the speed of sound, and $\tau_{C}$ is the phonon scattering relaxation time. In our fit we use $\theta_{D}=141 \mathrm{~K}$, estimated from the $C_{\mathrm{p}}$ data, and $v=2500 \mathrm{~m} / \mathrm{s}$, from mineral tetrahedrite having a similar density and bulk modulus [6] since no data exists for madocite. $\tau_{\mathrm{C}}^{-1}$ can be written as

$$
\tau_{C}^{-1}=\frac{v}{L}+A \omega^{4}+B \omega^{2} T \exp \left(-\frac{\theta_{D}}{3 T}\right)
$$

where $L$ is the grain size and the coefficients $A$ and $B$ are fitting parameters. The terms in Eq. (3) represent grain boundary scattering, point defect scattering, and Umklapp scattering, respectively. The fitting parameters, $L=1.2 \mu \mathrm{m}, \mathrm{A}=4.6 \times 10^{-42} \mathrm{~s}^{3}$ and $\mathrm{B}=3.7 \times 10^{-17} \mathrm{sK}^{-1}$, were uniquely defined using a minimization of the best sequence fit function compared to the data. These 
parameters resulted in excellent agreement with the experimental data, as shown in Figure 5.

The average grain size, $L$, is in agreement with the SEM results $(1.4 \mu \mathrm{m})$. The point defect scattering parameter $A$ is very small compared to other complex chalcogenides [23] and may be an indication of a relatively defect-free specimen, given the complex composition. The Umklapp scattering parameter $B$ is also relatively large compared with other chalcogenides and may be an indication of relatively high anharmonicity in this material.[23,24] No information is available on the Grüneisen parameter for this material, to the best of our knowledge, therefore the specific lattice dynamics for this material warrant further study.

\section{CONCLUSIONS}

We have synthesized polycrystalline $\mathrm{Pb}_{17}\left(\mathrm{Sb}_{0.75} \mathrm{As}_{0.25}\right)_{16} \mathrm{~S}_{41}$ by reaction of binary compounds followed by hot pressing. DTA data indicate a decomposition temperature of $668 \mathrm{~K}, C_{\mathrm{p}}$ data indicates $\theta_{D}=141 \mathrm{~K}$ and room temperature UV-Vis spectra indicate an optical band-gap of 1.32 $\mathrm{eV}$. The measured $\kappa$ values are low over a wide temperature range and are reported here for the first time. The relatively large bandgap precludes any potential for thermoelectric applications; however, allowing on the chalcogenide site with selenium or tellurium will presumably reduce this bandgap, as well as potentially further reduce $\kappa$ in this material system.

\section{ACKNOWLEDGEMENTS}

This work was supported by the National Science Foundation under Contract No. DMR1400957. KW acknowledges support by the II-VI Foundation Block-Gift Program. We acknowledge Dr. James R. Salvador for high temperature thermal diffusivity measurements. 


\section{References}

[1] T.P. Wada, S. Nakamura, T. Maeda, Prog. Photovolt: Res. Appl. 20 (2012) 520-525.

[2] S. Chen, X.G. Gong, C. Duan, Z. Zhu, J. Chu, A, Walsh, Y. Yao, J. Ma, S. Wei, Phys. Rev. B 83 (2011) 245202.

[3] G.S. Nolas, J. Sharp, H.J. Goldsmid, Thermoelectrics: Basic Principles and New Materials Developments, Springer, New York, 2001.

[4] X. Lu, D.T. Morelli, Phys. Chem. Chem. Phys. 15 (2013) 5762.

[5] Y. Dong, H. Wang, G.S. Nolas, Phys. Status Solidi RRL 8 (2014) 61.

[6] X. Lu, D.T. Morelli, Y. Xia, F. Zhou, V. Ozolins, H. Chi, X. Zhou, C. Uher, Adv. Energy Mater. 3 (2013) 342.

[7] P. Liu, T. Zhang, Y. Qiu, X. Shi, L. Chen, Energy Environ. Sci. 7 (2014) 4000.

[8] M. Wuttig, N. Yamada, Nature Mater. 6 (2007) 824.

[9] T. Siegrist, P. Merkelbach, M. Wuttig, Annu. Rev. Condens. Matter Phys. 3 (2012) 215.

[10] E. S. Toberer, L. L. Baranowski, C. Dames, Annu. Rev. Mater. Res. 42 (2012) 179.

[11] J. Callaway, Phys. Rev. 113 (1959) 1046-1051.

[12] J. Martin, S. Erickson, G.S. Nolas, P. Alboni, T.M. Tritt, J. Yang, J. Appl. Phys. 99 (2006) 044903.

[13] J. Martin, G.S. Nolas, Rev. Scientific Instruments 87 (2016) 015105/1-015105/8.

[14] J.W. Anthony, R.A. Bideaux, K.W. Bladh, M.C. Nichols, (1990) Handbook of Mineralogy: Volume 1: Elements, Sulfides, Sulfosalts, and references therein.

[15] D.S. Walia, L.L.Y. Chang, Can. Mineral. 12 (1973) 113-119. 
[16] U. Aydemir, C. Candolfi, H. Bormann, M. Baitinger, A. Ormeci, W. Carillo-Cabrera, C. Chubilleau, B. Lenoir, A. Dauscher, N. Oeschler, F. Steiglich, Yu. Grin, Dalton Trans. 39 (2010) 1078-1088.

[17] Y. Singh, Y. Lee, S. Nandi, A. Kreyssig, A. Ellern, S. Das, R. Nath, B.N. Harmon, A.I. Goldman, D.C. Johnston, Phys. Rev. B. 78 (2008) 104512/1-104512/7.

[18] Y. Luo, H. Han, H. Tan, X. Lin, Y. Li, S. Jiang, C. Feng, J. Dai, G. Cao, Z. Xu, S. Li, J. Phys.: Condens. Matter 23 (2011) 175701.

[19] H. He, W. Miiller, M.C. Aronson, Inorg. Chem. 53 (2014) 9115-9121.

[20] S. Stefanoski, J. Martin, G.S. Nolas, J. Phys.: Condens. Matter 22 (2010) 485404.

[21] C. Kittel, Introduction to Solid State Physics, $7^{\text {th }}$ edition, New York, 1996.

[22] K. Herrmann, in Hardness Testing: Principles and Applications; K. Herrmann, Eds.; ASM International, Materials Park, Ohio, 2011; Chapter 1.

[23] Y. Dong, K.Wei, G.S. Nolas, Phys. Rev. B. 87 (2013) 195203.

[24] K. Schotte, Zeitschrift fur Physik 196 (1966) 393. 


\section{Figure captions}

Figure 1. Measured and simulated XRD patterns of $\mathrm{Pb}_{17}\left(\mathrm{Sb}_{0.75} \mathrm{As}_{0.25}\right)_{16} \mathrm{~S}_{41}$. The inset is a schematic of the crystal structure.

Figure 2. TGA (solid) and DTA (dashed) data for $\mathrm{Pb}_{17}\left(\mathrm{Sb}_{0.75} \mathrm{As}_{0.25}\right)_{16} \mathrm{~S}_{41}$.

Figure 3. Room temperature UV-Vis spectra for $\mathrm{Pb}_{17}\left(\mathrm{Sb}_{0.75} \mathrm{As}_{0.25}\right)_{16} \mathrm{~S}_{41}$.

Figure 4. Temperature dependent $C_{\mathrm{p}}$ with the inset showing $C_{\mathrm{p}} / T$ versus $T^{2}$ data below $T=10 \mathrm{~K}$. The solid line in the inset is a fit by the expression $C_{\mathrm{p}} / T=\gamma+\beta T^{2}$.

Figure 5. Temperature dependent lattice thermal conductivity, $\kappa_{\mathrm{L}}$. The solid line is a fit to the data using the Debye model. 
Figures

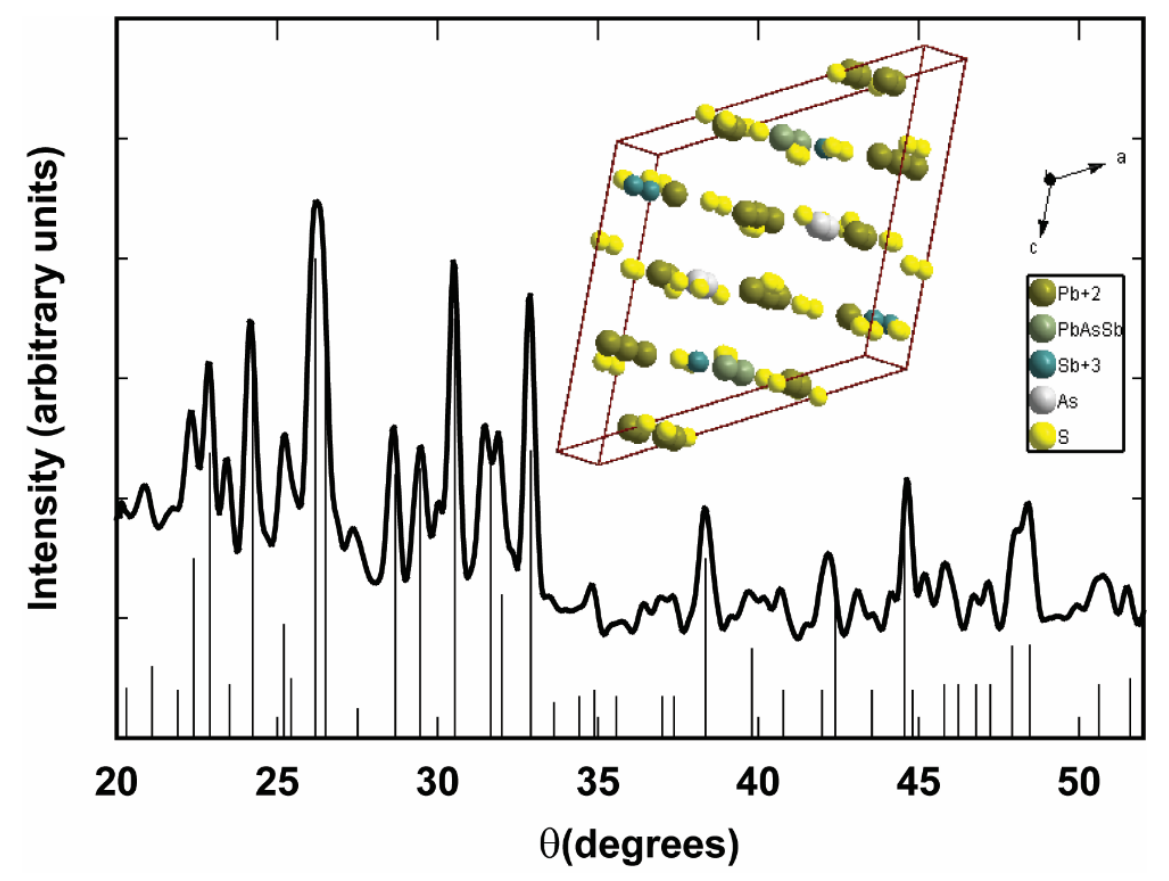

Figure 1. 


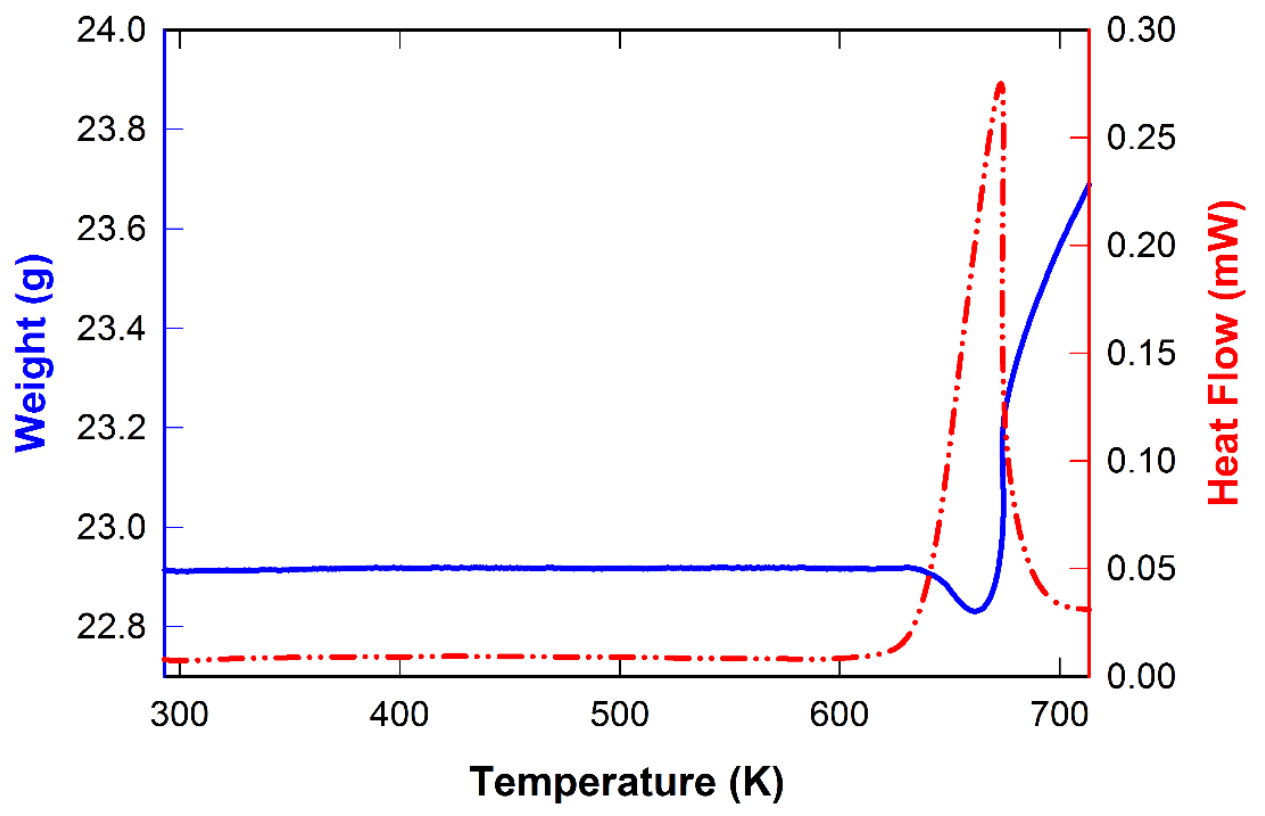

Figure 2. 


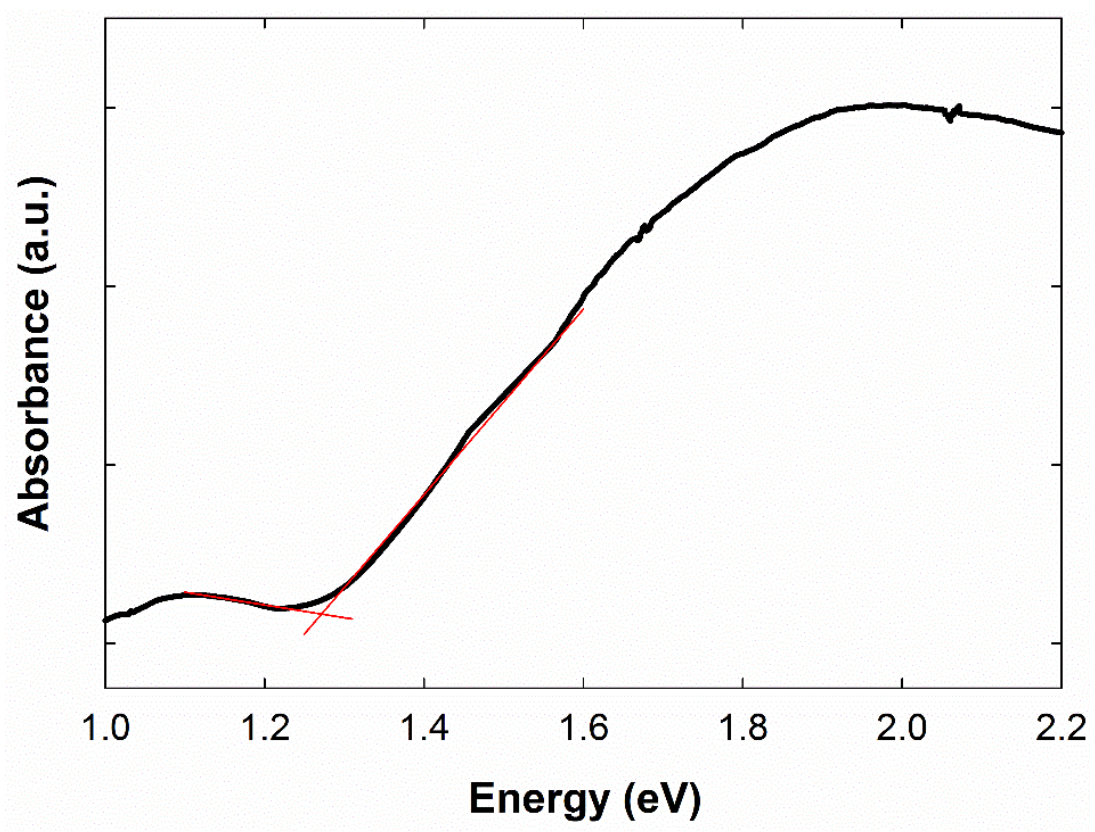

Figure 3. 


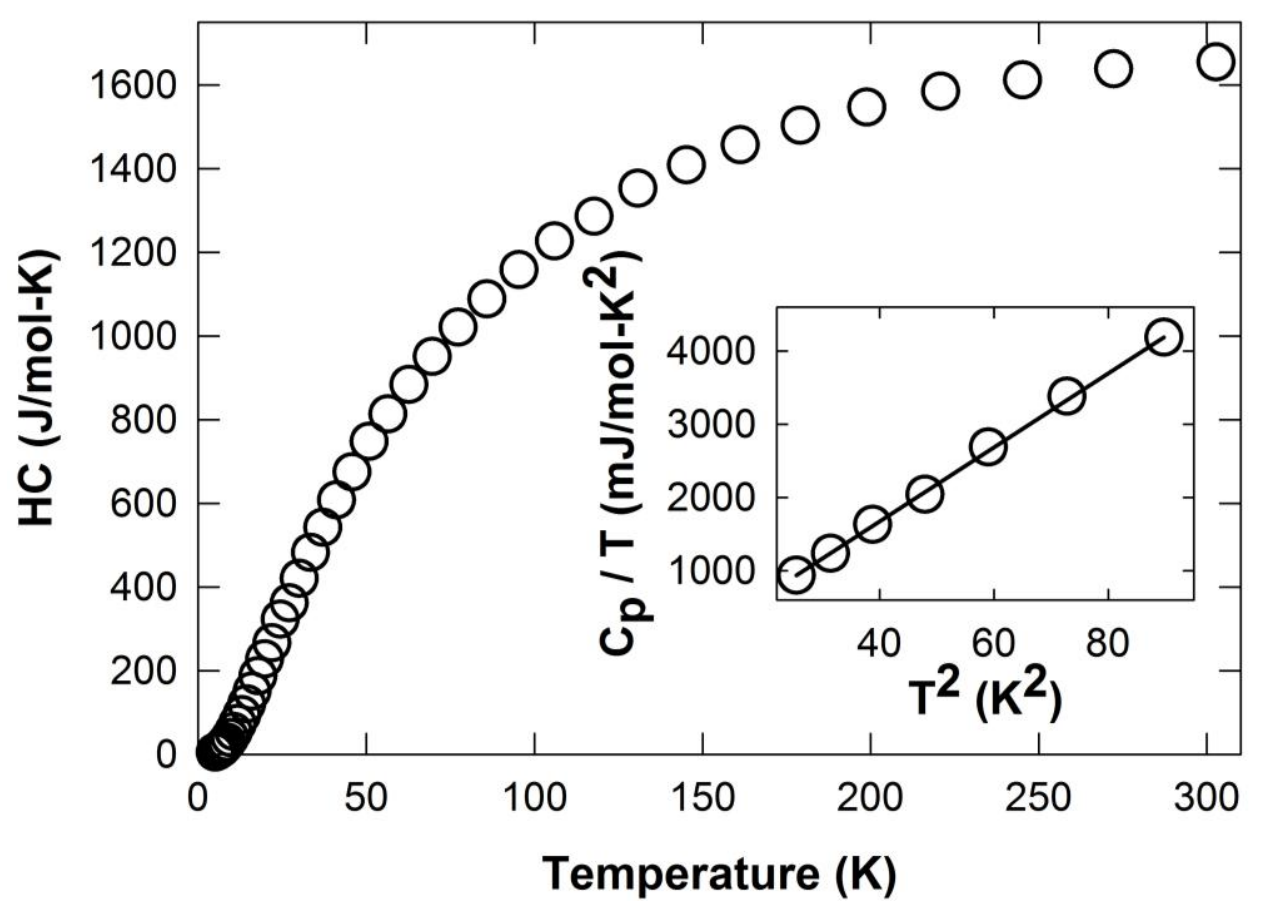

Figure 4. 


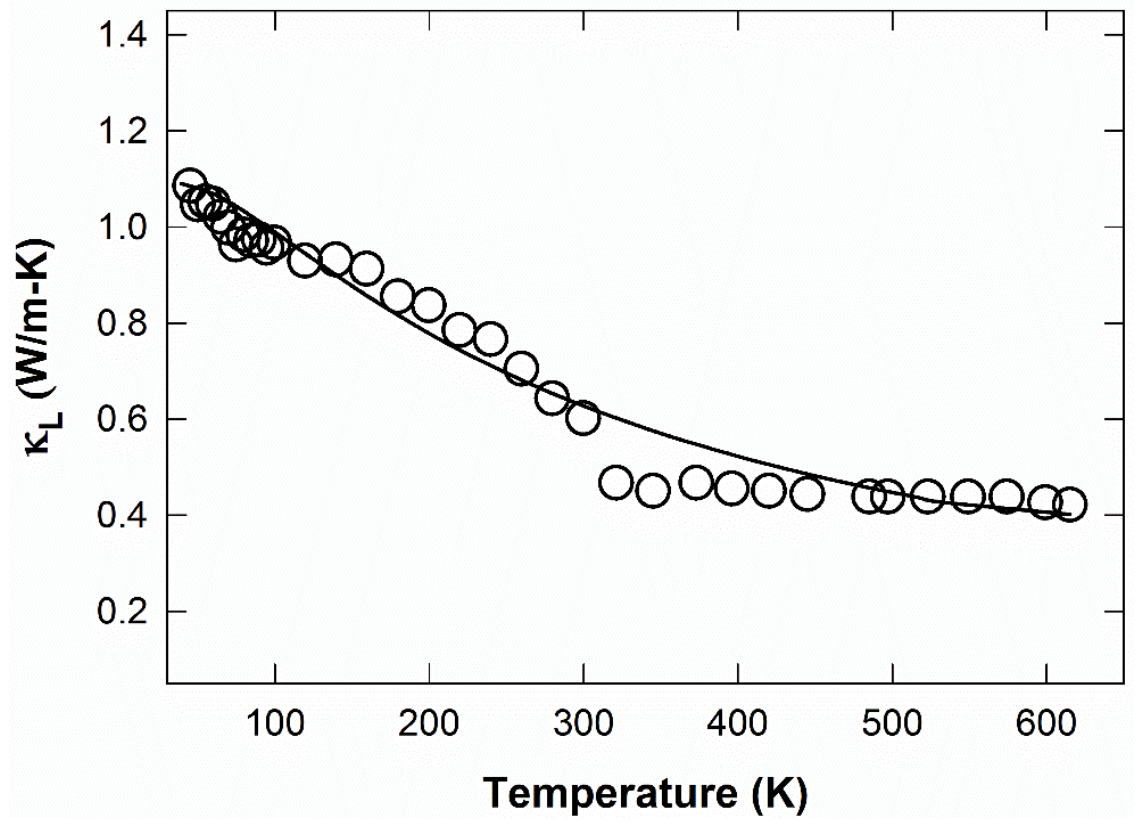

Figure 5. 


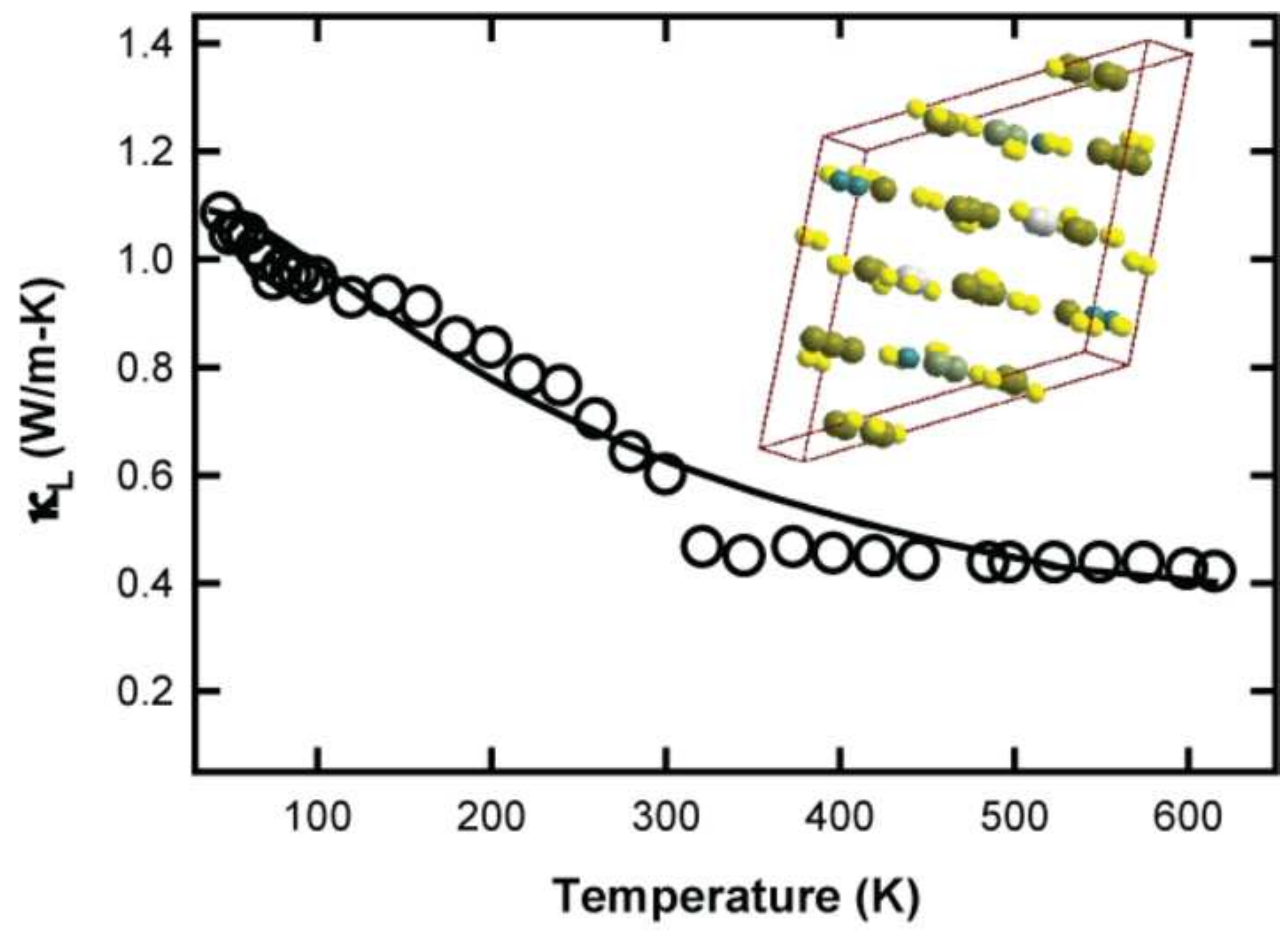

ical Abstract) 